\title{
Enhanced urinary odor discrimination in female aromatase knockout (ArKO) mice
}

\author{
Daniel W. Wesson ${ }^{\text {a }}$, Matthieu Keller ${ }^{\text {b }}$, Quentin Douhard ${ }^{\text {b }}$, Michael J. Baum ${ }^{\text {a }}$, Julie Bakker ${ }^{\text {b,* }}$ \\ a Department of Biology, Boston University, Boston, MA 02215, USA \\ ${ }^{\mathrm{b}}$ Center for Cellular and Molecular Neurobiology, University of Liège, Avenue de l'hopital 1 (B36), 4000 Liège (Sart Tilman), Belgium
}

Received 2 November 2005; revised 8 December 2005; accepted 13 December 2005

Available online 30 January 2006

\begin{abstract}
We asked whether odor discrimination abilities are sexually dimorphic in mice and, if so, whether the perinatal actions of estradiol contribute to these sex differences. The ability to discriminate different types of urinary odors was compared in male and female wild-type (WT) subjects and in mice with a homozygous-null mutation of the estrogen synthetic enzyme, aromatase (aromatase knockout; ArKO). Olfactory discrimination was assessed in WT and ArKO male and female mice after they were gonadectomized in adulthood and subsequently treated with estradiol benzoate. A liquid olfactometer was used to assess food-motivated olfactory discrimination capacity. All animals eventually learned to distinguish between urinary odors collected from gonadally intact males and estrous females; however, WT males as well as ArKO mice of both sexes learned this discrimination significantly more rapidly than WT females. Similar group differences were obtained when mice discriminated between urinary odors collected from gonadally intact vs. castrated males or between two non-social odorants, amyl and butyl acetate. When subjects had to discriminate volatile urinary odors from ovariectomized female mice treated with estradiol sequenced with progesterone versus estradiol alone, ArKO females quickly acquired the task whereas WT males and females as well as ArKO males failed to do so. These results demonstrated a strong sex dimorphism in olfactory discrimination ability, with WT males performing better than females. Furthermore, female ArKO mice showed an enhanced ability to discriminate very similar urinary odorants, perhaps due to an increased sensitivity of the main olfactory nervous system to adult estradiol treatment as a result of perinatal estrogen deprivation.
\end{abstract}

(C) 2005 Elsevier Inc. All rights reserved.

Keywords: Estrogens; Sex differences; Olfaction; Odor discrimination; Learning

Sex differences have been reported in olfactory sensitivity, with females being better able than males to detect male-derived odors. For instance, sows are significantly better than boars at using decreasing concentrations of the volatile male pig pheromone, androstenone, as a discriminative stimulus in operant tests for a sucrose reward (Dorries et al., 1995). In addition, using measurements of regional cerebral blood flow with positron emission tomography (Savic et al., 2001), women but not men show a significant activation of the preoptic area and ventromedial nuclei of the hypothalamus when actively smelling androstadienone, an androgenic compound secreted by the axillary glands in much higher concentrations in men than in women (Gower and Ruparelia, 1993). These sex differences in olfactory sensitivity are not only restricted to the detection of

\footnotetext{
* Corresponding author. Fax: +32 43665971 .

E-mail address: jbakker@ulg.ac.be (J. Bakker).
}

opposite sex odors, but also involve same-sex odors. For instance, using habituation/dishabituation tests to determine odor attraction thresholds, female mice respond more reliably than male mice to low concentrations of volatile urinary odors from either sex (Baum and Keverne, 2002; Pierman et al., 2006).

The existence of sex differences in olfactory sensitivity suggests that gonadal hormones may affect olfactory capabilities. The greater olfactory sensitivity observed in females may reflect differences in circulating sex steroids at the time of testing (activational effects) or a hard-wired sex dimorphism in the functioning of the olfactory system that resulted from the perinatal actions of gonadal hormones (organizational effects). The studies by Baum and Keverne (2002) and Pierman et al. (2006) were both conducted in long-term gonadectomized mice without any adult hormone treatment, suggesting that the observed sex differences in urine attraction thresholds reflect organizational and not activational effects of hormones on the 
olfactory system. Furthermore, male mice which were unable to produce any estrogens due to a targeted mutation in the aromatase gene (aromatase knockout or ArKO mice; Honda et al., 1998) show a female-typical capacity in detecting low concentrations of volatile urinary odors of either sex. This finding raises the possibility that the observed sex difference among normal mice in urine attraction thresholds resulted from the perinatal actions of estrogen in the male nervous system (Pierman et al., 2006). Likewise, Dorries et al. (1995) showed that the sex difference in pigs' ability to detect androstenone probably reflects a perinatal action of testosterone or estrogen in the male since the olfactory performance of neonatally castrated males to androstenone falls between those of sows and boars.

In contrast with olfactory sensitivity, much less is known about sex differences in olfactory discrimination abilities. One study (Mihalick, 2003) showed that early postnatal exposure to the synthetic estrogen, diethylstilbestrol, enhances olfactory discrimination learning in both male and female mice, although no sex differences were observed in untreated control animals. However, this study focused more on establishing the role of postnatal estrogens in the acquisition of the odor discrimination task than on the ability of mice to discriminate between various odors per se. The present study was designed to assess the effects of perinatal estrogen exposure on social odor discrimination by using the aromatase knockout mouse (ArKO) as a model. Previous work showed that ArKO mice of both sexes spend significantly less time than wild-type (WT) controls investigating volatile body odors from an intact male versus an estrous female in a Y-maze (Bakker et al., 2002a,b). These lower levels of olfactory investigation of volatile body odors are probably not due to an inability of ArKO mice to discriminate between male and female urinary odors since ArKO mice show clear dishabituation responses to successive presentations of undiluted male and female urinary odors in habituation/ dishabituation tests (Pierman et al., 2006). However, in the same study, ArKO females respond less reliably than WT controls when presented with a dilution series of estrous female urinary odors. Since habituation/dishabituation tests depend on the intrinsic motivation of animals to investigate the odors presented, it could not be determined whether the weaker behavioral performance of ArKO females reflected deficits in olfactory perception or in motivation. Therefore, in the present study, we used an operant food-motivated odor discrimination task to assess olfactory function of adult WT and ArKO males and females which had all been gonadectomized and treated in adulthood with a low dose of estradiol benzoate. We hypothesized that ArKO females would show deficient olfactory discrimination in light of their weaker performance observed in odor detection habituation/dishabituation tests (Pierman et al., 2006).

\section{Materials and methods}

\section{Animals}

ArKO mice were generated by targeted disruption of exons 1 and 2 of the Cyp 19 gene (Honda et al., 1998). Heterozygous males and females of the
$\mathrm{C} 57 \mathrm{Bl} / 6$ strain were bred to generate wild-type (WT) and homozygous-null (ArKO) offspring. Mice were genotyped by PCR analysis of tail DNA (for more detailed description, see Bakker et al., 2002a). Three weeks prior to olfactometer training, all animals were gonadectomized (GDX) under anesthesia induced by an intraperitoneal injection of a mixture of ketamine $(80 \mathrm{mg} / \mathrm{kg}$ per mouse) and medetomidine (Domitor, Pfizer, $1 \mathrm{mg} / \mathrm{kg}$ per mouse). Mice received atipamezole (antisedan, Pfizer, $4 \mathrm{mg} / \mathrm{kg}$ per mouse) subcutaneously at the end of the surgery to antagonize medetomidine-induced effects, thus accelerating their recovery. After gonadectomy, mice were placed into individual cages under a normal day light-dark cycle (12L:12D, lights on at 08:00 h). Since castration of male rats (in the absence of sex steroid replacement) had been shown to retard their acquisition of an olfactometer task (Doty and Ferguson-Segall, 1989), all experimental animals were given daily injections of $1 \mu \mathrm{g} 17 \beta$-estradiol benzoate (EB; ICN Biomedicals, Irvine, California) dissolved in sesame oil starting 2 weeks after GDX and 2 weeks prior to operant training so as to maximize their performance in the olfactometer.

All experiments were conducted in accordance with the guidelines of the National Institutes of Health and were approved by the Ethical Committee for Animal Use at the University of Liège and the Boston University Institutional Animal Care Committee.

\section{Urine collection}

Urine was collected into odorless vials from mice as they were held by the nape of their neck. Odorless vials were obtained by cleaning glass vials multiple times with $95 \%$ ethanol followed by multiple washes with distilled water. Intact male urine was collected from 12 adult gonadally intact C57Bl6 males. GDX male urine was collected from 12 adult C57Bl6 males, who were GDX at least 1 month prior to urine collection. Estrous female $(\mathrm{E}+\mathrm{P}$-female) urine was obtained from 15 ovariectomized C57Bl6 females implanted subcutaneously with a silastic capsule filled with crystalline $17 \beta$-estradiol (diluted 1:1 with cholesterol) and primed with progesterone (500 $\mu \mathrm{g} /$ mouse) $3 \mathrm{~h}$ prior to the collection. This hormone regimen has been shown to successfully induce behavioral estrus in female mice (Bakker et al., 2002a,b). Finally, estrogentreated female (E-female) urine was collected from the same 15 ovariectomized C57Bl6 females, without being injected with progesterone. Same-sex urine samples were pooled and stored in aliquots at $-20^{\circ} \mathrm{C}$.

\section{Food deprivation}

Mice were placed on a $24 \mathrm{~h}$ food deprivation schedule, beginning 1 week prior to testing, in order to motivate mice to perform the operant odor discrimination task with the palatable nutritional supplement, Pediasure (Ross pediatrics, Columbus, Ohio) serving as the reward. Each mouse typically received no more than $500 \mu 1$ Pediasure during a day's testing session. In addition to the Pediasure consumed during operant tests, all mice were given one food pellet a day (approximately 3-4 g) immediately after each day's testing session. Subjects were weighed daily to ensure that body weights did not decrease more than $20 \%$ from a pre-deprivation baseline. Water was available ad libitum except during actual testing.

\section{Olfactometry}

An automated liquid dilution 8-channel olfactometer (Knosys Ltd., Lutz, Florida) was employed for operant assessment of olfactory discrimination capacity. Subjects were placed in a Plexiglas test chamber equipped with a tube embedded in an odor-sampling port to deliver liquid Pediasure as a reward. A flow-chart outlining the operant task is shown in Fig. 1, and the olfactometer has been previously described by Bodyak and Slotnick (1999). The percent of correct responses was determined for each block of 20 trials (a total of 5 blocks were given during each daily test session). Notably, the odor concentration which the animal was presented within the sampling port was much lower than the volume/volume $(\mathrm{v} / \mathrm{v})$ concentration which was placed in the odor vials. This decrease in concentration is due to several factors (e.g., air flow rate, surface area of liquid odor in flask) and results in approximately $3 \%$ of the odor's $\mathrm{v} / \mathrm{v}$ concentration actually passing the nose of the animal as it samples in the port (Bodyak and Slotnick, 1999). For example, an odor flask holding a $10^{-3}$ 
Nose poke into odor sampling port breaks photobeam

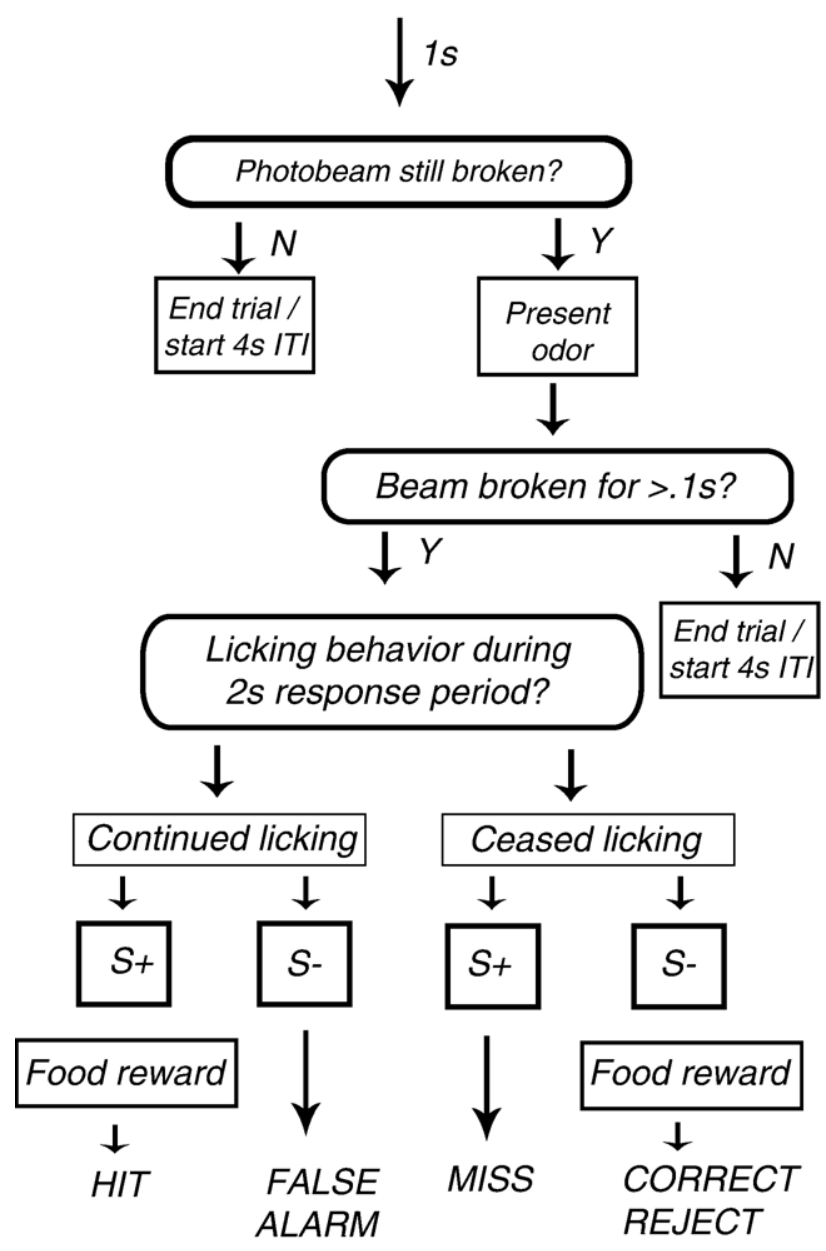

Fig. 1. Flow-chart outlining the procedure for assessing odor discrimination capacity using a liquid olfactometer. Briefly, the olfactometer presents an odor when the mouse breaks a photobeam with its snout. When the photobeam is broken, a stream of purified air displaces the odor headspace in the odor vials, sending this odor through a mixing manifold to pass by the nose of the animal as it licks from a reward spout. In this go, no-go discrimination paradigm, mice were rewarded with approximately $3 \mu 1$ of Pediasure when they responded correctly by licking to the presentation of the $\mathrm{S}+$ (hit) odor. If the animal did not lick for presentation of the $\mathrm{S}+$ odor (miss) or did lick for presentations of the $\mathrm{S}-$ odor (false alarm), it was given a negative score. Alternatively, in cases when the animal did not lick for presentations of the $\mathrm{S}$ - odor (correct reject), the animal received a positive score. ITI: inter-trial interval.

$(1: 1000, \mathrm{v} / \mathrm{v})$ solution actually results in a $3 \times 10^{-5}$ odor concentration reaching the animal during testing. In this experiment, all concentrations will be expressed in terms of the actual liquid concentration $(\mathrm{v} / \mathrm{v})$ in the odor flask.

Operant training started approximately 2 weeks after the onset of EB treatment and 1 week after initiating food deprivation. Mice were first trained on an odor detection task for $10^{-3}(\mathrm{v} / \mathrm{v})$ amyl acetate (S+, MD Biomedicals, Irvine, California) versus mineral oil ( $\mathrm{S}-$ ). After acquiring this task, defined by a response criterion of $85 \%$ correct in 2 consecutive blocks of 20 trials, the animals were switched to intact male urine $\left(10^{-2} \mathrm{v} / \mathrm{v}\right)$ as the $\mathrm{S}+$ and water as the $\mathrm{S}-$. In order for animals to proceed into data collection tests, they had to meet the criterion of $85 \%$ correct in 2 consecutive blocks of 20 trials for both the amyl acetate and the intact male urine training sessions. For data collection, we presented the subjects with four novel tasks, three of which involved discriminating between urinary social odors $\left(10^{-2} \mathrm{v} / \mathrm{v}\right)$ and one that involved discriminating between two non-social odorants $\left(10^{-3}\right.$ amyl acetate ( $\left.\mathrm{S}+\right)$ versus $n$-butyl acetate (S-, MD Biomedicals, Irvine, California). For the social odor discrimination tasks, animals were first asked to discriminate intact male urine $(\mathrm{S}+)$ from $\mathrm{E}+\mathrm{P}$-female urine $\left(\mathrm{S}^{-}\right)$, then intact male urine $(\mathrm{S}+)$ from GDX male urine ( $\mathrm{S}-$ ), and, finally, $\mathrm{E}+\mathrm{P}$-female urine $(\mathrm{S}+)$ from urine of E-females $(\mathrm{S}-$ ) The tests for social odors were presented in the aforementioned order followed by the tests for non-biological odors. Thus, animals were tested first with what was hypothesized to be the most potent, and thus simplest, social odor task, i.e., male vs. female urinary odors, followed by same-sex urine tasks with the hypothesis that the intact male vs. the GDX male task would be the easiest for the female subjects and the E + P-female vs. E-female task easiest for the male subjects. The amyl acetate vs. butyl acetate choice was chosen as the final discrimination task to gauge the olfactory discrimination abilities of all animals on a task that was non-social in nature. For simple testing feasibility, the order of odor testing was not randomized between subjects nor between groups. All discrimination tasks allowed the animal 100 trials to acquire each olfactory discrimination (half $\mathrm{S}+$ and half $\mathrm{S}-$ ). The concentrations of the social odors and non-social odors that we chose for the discrimination paradigms are based on pilot work showing that animals could readily acquire and complete a task detecting urine at a $10^{-2}$ concentration and amyl acetate and butyl acetate at a $10^{-3}$ concentration. Similar concentrations of acetates have been used in past olfactometric work on mice (Bodyak and Slotnick, 1999).

After all odor discrimination tests had been completed, animals were fooddeprived for $24 \mathrm{~h}$ and evaluated for food motivation. The animals were individually allowed access to $3 \mu 1$ reinforcements of Pediasure in the olfactometer's operant box for $10 \mathrm{~min}$ with a $1 \mathrm{~s}$ inter-trial interval. The total number of reinforcements was recorded as an indicator of food motivation and compared across groups to ensure that no one group's olfactory discrimination performance in this food motivation-based test reflected a low level of hunger

Data which were collected in QBASIC during behavioral testing by the olfactometer were imported into StatView v5.0 (Abacus Concepts Inc., Berkeley, CA) for analysis. The percentage of correct responses for subjects in each of the 4 different odor discrimination tasks were analyzed using 2-way ANOVAs for independent groups (4) and repeated measures (5 blocks of 20 trials). The mean number of false alarms made during testing, i.e., when the animal licked for the S- stimulus, was also calculated for each group for each odor discrimination task and analyzed using one-way ANOVAs for independent groups (4). Post-hoc group comparisons after a significant ANOVA result were made using Fishers PLSD tests. Food motivation scores were also analyzed with ANOVA and Fishers PLSD post-hoc test.

\section{Results}

As seen in Fig. 2A, WT males as well as ArKO males and females acquired the discrimination between gonadally intact male and $\mathrm{E}+\mathrm{P}$ female urinary odors more quickly than WT females. There was an overall group effect $(F(3,15)=7.41$, $P=0.0028)$ and a significant overall repeated measures effect $(F(4,60)=19.85, P<0.0001)$, but no significant interaction, in subjects' discrimination of intact male vs. E + P-female urine. Post-hoc analysis of the group effect showed that WT males as well as ArKO mice of both sexes had better scores than WT females and that ArKO females, in turn, had better scores than ArKO males. The significant effect of repeated measures indicated that all groups were able to discriminate between male and female urinary odors by the end of testing. The poor performance of WT females was primarily due to false alarms, that is, they licked when the $\mathrm{S}$ - was presented (the male odors in this case; Table 1). One-way ANOVA on false alarm frequencies showed a significant effect of group $(F(3,14)=6.95, P=0.004)$. Post-hoc comparisons revealed that false alarm frequencies were higher in WT females compared to WT males and ArKO females, but not to ArKO males. 
A Intact male urine (S+) E+P-female urine (S-)
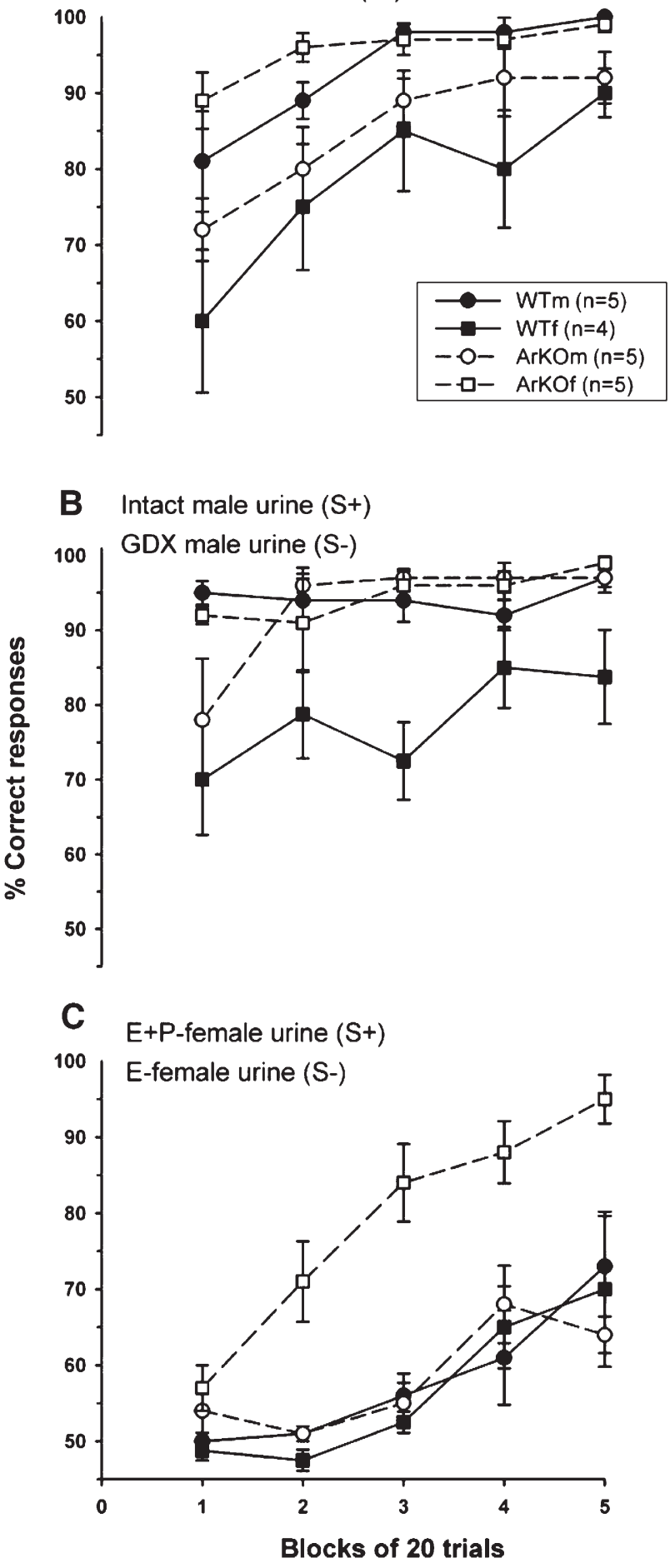

Fig. 2. Ability of ArKO and WT male and female mice to learn to discriminate between gonadally intact male and estrous $(\mathrm{E}+\mathrm{P})$ female urine $(\mathrm{A})$, gonadally intact and castrated male urine (B), or between $\mathrm{E}+\mathrm{P}$-female and E-female urine (C). $\mathrm{S}+=$ rewarded stimulus; $\mathrm{S}-=$ non-rewarded stimulus. Data are expressed as mean $\pm \mathrm{SEM}$.

When mice discriminated between intact male versus GDX male urine, all groups except for WT females quickly acquired this task (Fig. 2B). There was a significant overall group effect $(F(3,15)=8.81, P=0.0013)$, a repeated measures effect $(F(4,60)=5.78, P=0.0005)$, as well as a significant interaction $(F(12,60)=2.05, P=0.035)$. Post-hoc analyses of the group effect showed that WT males, ArKO males, and ArKO females were consistently better at discriminating this task than WT females. Further analyses of the interaction revealed that ArKO males were somewhat slower than WT males and ArKO females but not WT females, in acquiring this task. Again, the lower scores of WT females were primarily due to making more false alarms (Table 1). One-way ANOVA on false alarm frequencies showed a significant group effect $(F(3,14)=6.14$, $P=0.007)$. Post-hoc analysis showed that WT females made more false alarms than WT and ArKO males and females.

As seen in Fig. 2C, the ArKO females were the only group to ever reach criterion performance $(\geq 85 \%$ correct responses) discriminating $\mathrm{E}+\mathrm{P}$-female urine from $\mathrm{E}$-female urine. Neither WT male and female nor ArKO male mice learned to discriminate these two urinary odors. This was confirmed by ANOVA which showed an overall group effect $(F(3,15)=14.09, P=0.0001)$, an effect of repeated measures $(F(4,60)=32.50, P<0.0001)$, and a significant interaction between those factors $(F(12,60)=2.44, P=0.012)$. Post-hoc analysis of the group effect showed that ArKO females had better scores than all other groups, whereas the latter did not differ among each other. Post-hoc analysis of the interaction showed that only in ArKO females did the percentage of correct responses increase over repeated trials. No such significant increase was observed in the other groups. These group differences in performance were again due to differences in number of false alarms (Table 1). One-way ANOVA showed a significant effect of group $(F(3,14)=7.41$, $P=0.003$ ). Post-hoc analysis revealed that ArKO females made fewer false alarms than WT males and females or ArKO males.

In the non-social odor discrimination task of amyl acetate versus butyl acetate (Fig. 3), all groups of animals except for the WT females readily acquired the task $(\geq 85 \%$ correct responses). This large difference between the WT females and all other groups was reflected in a significant overall group effect $(F(3,15)=7.66, P=0.0025)$ and a significant repeated

Table 1

False alarm frequencies during odor discrimination tasks

\begin{tabular}{lcccc}
\hline \multirow{2}{*}{$\begin{array}{l}\text { Odors } \\
\text { discriminated }\end{array}$} & Group & & \\
\cline { 2 - 5 } & WT-M (5) & \multicolumn{1}{c}{ WT-F (3) } & ArKO-M (5) & ArKO-F (5) \\
\hline M vs. F & $6.6 \pm 0.9$ & $21.67 \pm 6.9^{\mathrm{b}}$ & $13.8 \pm 2.5$ & $4 \pm 1.6$ \\
M vs. GDX-M & $4.6 \pm 0.5$ & $20.3 \pm 5.8^{\mathrm{c}}$ & $7 \pm 2.6$ & $5.2 \pm 1.9$ \\
E-F vs. E + P-F & $39.6 \pm 4.8^{\mathrm{d}}$ & $39.3 \pm 3.2^{\mathrm{d}}$ & $38.8 \pm 1.7^{\mathrm{d}}$ & $20.8 \pm 3.2$ \\
AA vs. BA & $10.6 \pm 2.4$ & $34.3 \pm 7.2^{\mathrm{c}}$ & $14.6 \pm 3$ & $15 \pm 3.76$
\end{tabular}

Values indicate mean false alarm responses \pm SEM. The number of animals in each group is indicated in parentheses.

Abbreviations: $\mathrm{AA}=$ amyl acetate; $\mathrm{ArKO}=$ aromatase knockout; $\mathrm{BA}=$ butyl acetate; $\mathrm{E}=$ estradiol $; \mathrm{F}=$ female $; \mathrm{GDX}=$ gonadectomized; $\mathrm{M}=$ male; $P=$ progesterone; $\mathrm{WT}=$ wild-type.

${ }^{\text {a }}$ Data from one WT female could no longer be retrieved from the hard-drive.

b $P<0.05$ compared to WT males and ArKO females.

${ }^{c} P<0.05$ compared to WT males and ArKO males and females.

d $P<0.05$ compared to ArKO females. 


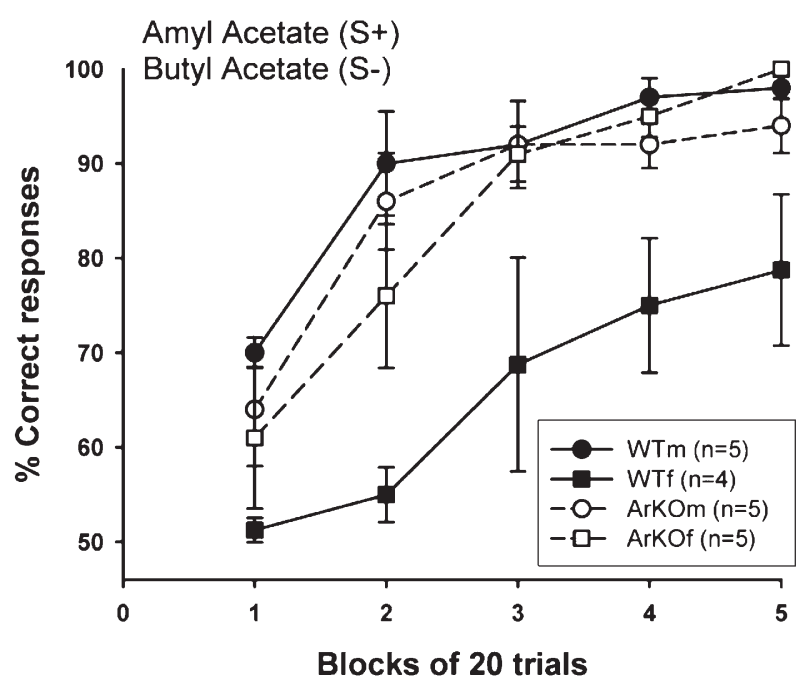

Fig. 3. Ability of ArKO and WT male and female mice to learn to discriminate between amyl and butyl acetate. $\mathrm{S}+=$ rewarded stimulus; $\mathrm{S}-=$ non-rewarded stimulus. Data are expressed as mean \pm SEM.

measures effect $(F(4,60)=43.96, P<0.0001)$, but no significant interaction. Post-hoc analysis of the group effect showed that WT males as well as ArKO mice of both sexes had better scores than WT females. The repeated measures effect in the absence of a significant interaction indicated that all groups showed a similar acquisition of this task. Again, the lower scores in WT females were due to making more false alarms than the other groups (Table 1). One-way ANOVA showed a significant effect of group $(F(3,14)=6.08, P=0.007)$. Post-hoc analysis showed that WT females made more false alarms than any other group.

Results from the food motivation task showed no statistically significant group differences in the number of food reinforcements taken within the 10-min testing period (ANOVA with Fishers PLSD, $P \geq 0.2733$ ). The mean number of food reinforcements and their standard errors were $313 \pm 32$ for the WT females, $399 \pm 59$ for the WT males, $359 \pm 43$ for ArKO females, and $409 \pm 26$ for ArKO males.

\section{Discussion}

The present study showed a strong sex dimorphism in olfactory discrimination abilities, with WT males being more proficient than WT females at discriminating between several types of urinary odors (i.e., male versus estrous female; gonadally intact versus castrated male) as well as between two non-social odors, amyl versus butyl acetate, in a foodmotivated operant task. Surprisingly, ArKO females resembled WT males in their ability to discriminate these 3 pairs of odorants. Even more striking was the ability of ArKO females to discriminate between urinary odors derived from ovariectomized female mice previously treated with estradiol sequenced with progesterone versus estradiol alone. Neither WT male nor female nor ArKO male mice learned to discriminate these two urinary odors which presumably are chemically very closely related. The performance of ArKO males was generally very similar to that of WT males with the exception of the discrimination task involving male versus female urinary odors for which their performance fell between that of WT males and females.

The finding that males were superior to females in discriminating between various odors may indicate the presence of general sex differences in the ability to perform in operant tasks. Some evidence for female mice learning more slowly than males comes from the study by Mishima et al. (1986). When asked to lever press for access to a food cup, fooddeprived males acquired the task more quickly than females. Likewise, using an 8-arm radial maze, males made significantly fewer errors than females in finding food at the end of the baited arms. In the present study, female WT mice seem to acquire the discrimination tasks more slowly than male WT mice. Thus, it cannot be excluded that most effects observed here reflect differences in learning abilities rather than differences in olfactory perception. For instance, it is possible that WT males and females, as well as ArKO males, would eventually have learned to discriminate between $\mathrm{E}$ - and $\mathrm{E}+\mathrm{P}$-female urine if more trials had been run.

The present results are in contrast with previous results obtained using habituation/dishabituation tests (Pierman et al., 2006). In those experiments, ArKO mice of both sexes reliably distinguished between undiluted volatile urinary odors of either adult males or estrous females versus deionized water as well as between these urinary odors themselves. However, ArKO mice of both sexes were less motivated than WT controls to investigate same-sex odors when they were presented last in the sequence of stimuli. Furthermore, female ArKO mice responded less reliably when presented with a dilution series of estrous female urine. Female ArKO mice failed to show significant dishabituation responses to two (1:20 and 1:80) dilutions of female urine, perhaps again, because of a reduced motivation to investigate less salient, same-sex urinary odors (Pierman et al., 2006). Since the habituation/dishabituation test paradigm depends on the intrinsic motivation of the animal to respond to the odor stimuli presented, it could not be determined whether the deficient behavioral responses of ArKO females to estrous female urine were due to motivational or olfactory perceptual deficits. Therefore, a food-motivated olfactory discrimination task was used in the present study to assess olfactory function in ArKO females. Since ArKO females did not show an increased motivation to consume the Pediasure liquid food in the operant chamber compared to the other groups, the heightened performance of ArKO females in the olfactometer tests presumably reflected an enhanced capacity of ArKO females to discriminate between very similar urinary odors. This outcome suggests that their weak performance in the habituation/dishabituation tests of odor detection (Pierman et al., 2006) cannot be attributed to deficits in function of the main olfactory system. Thus, the previous finding (Bakker et al., 2002a,b) that ArKO mice of both sexes spent less time investigating volatile body odors from either an intact male or an estrous female in a Y-maze most likely did not reflect deficits in their ability to detect or discriminate between these 
odors but rather a reduced motivation to actively investigate these odors.

Paradoxically, ArKO females resembled WT males in their performance of several odor discrimination tasks, suggesting that their olfactory discrimination abilities may have been masculinized. However, it is quite unlikely that ArKO females have been masculinized during perinatal development due to excessive exposure to androgens (since they are not able to aromatize androgenic precursor into an estrogen; Bakker et al., 2004b). First, the fetal and neonatal rodent ovary does not produce sex steroids (Lamprecht et al., 1976; Weniger et al., 1993), making it unlikely that there is an accumulation of androgenic substrate in ArKO females. Second, ArKO females showed very low levels of male-typical sexual behavior in adulthood following treatment with testosterone and estradiol (Bakker et al., 2002a,b). In fact, ArKO females showed less male-typical sexual behavior than WT females. This latter observation clearly argues against a masculinization of the ArKO female brain. In this respect, it is interesting to note that our work in male ArKO mice (Bakker et al., 2002a, 2004a) and the work of Sato et al. (2004) using mice with a null mutation in the androgen receptor clearly show that the masculinization of the mouse brain requires the presence of androgens as well as a functional androgen receptor. Sato et al. (2004) showed that WT females which were treated pre- and postnatally (from embryonic day 14 until postnatal day 8) with dihydrotestosterone (DHT) showed higher levels of mounting and intromissive behaviors, and at times even ejaculatory patterns, than untreated controls. This masculinization of the brain by perinatal DHT treatment was absent in male and female mice carrying a null mutation of the androgen receptor (Sato et al., 2004). These results also show that the critical period for brain masculinization does not extend beyond the early neonatal period (i.e., day 8 after birth) which is well before the time that mouse ovaries start to produce androgenic precursor. Taken together, it seems unlikely that the enhanced odor discrimination ability of ArKO females can be attributed to brain masculinization induced by excessive androgen exposure during perinatal development.

The superior olfactory performance of ArKO females over WT females may have resulted from the low level of estrogenic stimulation coupled with the absence of any male-typical features of genetic or steroidal signaling sustained perinatally. Previous work (Agarwal et al., 2000) showed a 2-fold increase in forebrain estrogen receptor- $\alpha$ content in adult ArKO compared to WT mice, indicating that estrogen normally down-regulates the expression of estrogen receptor- $\alpha$. Longterm deprivation of estrogenic stimulation also was found to upregulate (4- to 10-fold increase) estrogen receptor- $\alpha$ as well as MAP kinase, phophoinositol 3 kinase (Pi3K) growth factor expression in breast cancer cells (Santen et al., 2005). As a result, an enhanced sensitivity to the proliferative effects of estrogen treatment was observed. These findings thus raise the question of whether ArKO females in the present study were more sensitive than WT controls to any activational effect of estradiol treatment on olfactory discrimination capacity. It is puzzling that we did not observe heightened olfactory discrimination in ArKO males, which presumably also were deprived of estrogenic stimulation before the onset of the experiment. Perhaps perinatal sex differences in testosterone's action and/or sex chromosome gene expression account for the discrepancy between ArKO male and female mice in their olfactory responses to estradiol treatment in adulthood. At present, there is no reason to believe that male ArKO mice do not undergo a normal masculinization of the brain. However, in contrast with female ArKO and WT mice, considerable levels of testosterone circulate in male mice during early development (Pointis et al., 1980). It is thus plausible that ArKO males have been exposed to increased androgenic action compared to WT males since androgens were not aromatized into estrogens, which may have compromised their later responses to adult estradiol treatment.

There is an expanding literature showing that genes on the sex chromosomes may contribute to the development of certain sex differences in the brain (Arnold, 2004). In this respect, it is interesting to note that recent evidence suggests that genes expressed off the sex chromosomes may directly control sex dimorphisms in the display of mouse aggression as well as of parental behaviors (E.F. Rissman, personal communication). Thus, it is possible that the differences between ArKO male and female mice (such as the observed difference between ArKO females and males in their ability to make a difficult urinary odor discrimination) reflect differences in sex chromosome gene expression.

\section{Acknowledgments}

This work was supported by NICHD grant No. HD044897 to $\mathrm{JB}$ and MJB and a grant from the Fonds National de la Recherche Scientifique (No. 1.5.082.04) to JB. J. Bakker is a Research Associate from the Fonds National de la Recherche Scientifique.

\section{References}

Agarwal, V.R., Sinton, C.M., Liang, C., Fisher, C., German, D.C., Simpson, E.R., 2000. Upregulation of estrogen receptors in the forebrain of aromatase knockout (ArKO) mice. Mol. Cell. Endocrinol. 162, 9-16.

Arnold, A.P., 2004. Sex chromosomes and brain gender. Nat. Rev., Neurosci. 5, 701-708.

Bakker, J., Honda, S., Harada, N., Balthazart, J., 2002a. Sexual partner preference requires a functional aromatase (cyp19) gene in male mice. Horm. Behav. 42, 158-171.

Bakker, J., Honda, S., Harada, N., Balthazart, J., 2002b. The aromatase knockout mouse provides new evidence that estradiol is required during development in the female for the expression of sociosexual behaviors in adulthood. J. Neurosci. 22, 9104-9112.

Bakker, J., Honda, S., Harada, N., Balthazart, J., 2004a. Restoration of male sexual behavior by adult exogenous estrogens in male aromatase knockout mice. Horm. Behav. 46, 1-10.

Bakker, J., Baillien, M., Honda, S., Harada, N., Balthazart, J., $2004 b$. Relationships between aromatase activity in the brain and gonads and behavioural deficits in homozygous and heterozygous aromatase knockout mice. J. Neuroendocrinol. 16, 483-490.

Baum, M.J., Keverne, E.B., 2002. Sex difference in attraction thresholds for volatile odors from male and estrous female mouse urine. Horm. Behav. 41, 213-219. 
Bodyak, N., Slotnick, B., 1999. Performance of mice in an automated olfactometer: odor detection, discrimination and odor memory. Chem. Senses 24, 637-645.

Dorries, K.M., Adkins-Regan, E., Halpern, B.P., 1995. Olfactory sensitivity to the pheromone, androstenone, is sexually dimorphic in the pig. Physiol. Behav. 57, 255-259.

Doty, R.L., Ferguson-Segall, M., 1989. Influence of adult castration on the olfactory sensitivity of the male rat: a signal detection analysis. Behav. Neurosci. 103, 691-694.

Gower, D.B., Ruparelia, B.A., 1993. Olfaction in humans with special reference to odorous 16-androstenes: their occurrence, perception and possible social, psychological and sexual impact. J. Endocrinol. 137, 167-187.

Honda, S., Harada, N., Ito, S., Takagi, Y., Maeda, S., 1998. Disruption of sexual behavior in male aromatase-deficient mice lacking exons 1 and 2 of the cyp19 gene. Biochem. Biophys. Res. Commun. 252, 445-449.

Lamprecht, S.A., Kohen, F., Ausher, J., Zor, U., Lindner, H.R., 1976. Hormonal stimulation of oestradiol-17 beta release from the rat ovary during early postnatal development. J. Endocrinol. 68, 343-344.

Mihalick, S.M., 2003. Perinatal exposure to diethylstilbestrol improves olfactory discrimination learning in male and female Swiss-Webster mice. Neurobiol. Learn. Mem. 80, 55-62.

Mishima, N., Higashitani, F., Teraoka, K., Yoshioka, R., 1986. Sex differences in appetitive learning of mice. Physiol. Behav. 37, 263-268.
Pierman, S., Douhard, Q., Balthazart, J., Baum, M.J., Bakker, J., 2006. Attraction thresholds and sex discrimination of urinary odorants in male and female aromatase knockout (ArKO) mice. Horm. Behav. 49, 96-104.

Pointis, G., Latreille, M.T., Cedard, L., 1980. Gonado-pituitary relationships in the fetal mouse at various times during sexual differentiation. J. Endocrinol. $86,483-488$.

Santen, R.J., Song, R.X., Zhang, Z., Kumar, R., Jeng, M.-H., Masamura, S., Lawrence Jr., J., MacMahon, L.P., Yue, W., Bernstein, L., 2005. Adaptive hypersensitivity to estrogen: mechanisms and clinical relevance to aromatase inhibitor therapy in breast cancer treatment. J. Steroid Biochem. Mol. Biol. 95, 155-165.

Sato, T., Matsumoto, T., Kawano, H., Watanabe, T., Uematsu, Y., Sekine, K., Fukuda, T., Aihara, K., Krust, A., Yamada, T., Nakamichi, Y., Yamamoto, Y., Nakamura, T., Yoshimura, K., Yoshizawa, T., Metzger, D., Chambon, P., Kato, S., 2004. Brain masculinization requires androgen receptor function. Proc. Natl. Acad. Sci. U. S. A. 101, 1673-1678.

Savic, I., Berglund, H., Gulyas, B., Roland, P., 2001. Smelling of odorous sex hormone-like compounds causes sex-differentiated hypothalamic activations in humans. Neuron 31, 661-668.

Weniger, J.P., Zeis, A., Chouraqui, J., 1993. Estrogen production by fetal and infantile rat ovaries. Reprod. Nutr. Dev 33, 129-136. 